\title{
B-type natriuretic peptide and renovascular hypertension; is there any relation?
}

\author{
Authors: \\ Shokoufeh HAJSADEGHI ${ }^{1}$, Yaghoub BAGHERI ${ }^{2}$, Mohammad Javad MANTEGHI ${ }^{3}$, \\ Ata FIROUZI ${ }^{4}$, Scott Reza JAFARIAN KERMAN ${ }^{5}$, Morteza HASSANZADEH ${ }^{6}$, \\ 1. Associate professor of Cardiology, Echocardiography Fellow, Research Center for Prevention \\ of Cardiovascular Disease, Institute of endocrinology \& metabolism, Iran University of \\ Medical Sciences, Tehran, Iran \\ 2. Cardiologist, Shahid Rajaei Heart Hospital, Iran University of Medical Sciences, Tehran, Iran \\ 3. Medical Student Research Committee, Faculty of Medicine, Iran University of Medical \\ Sciences, Tehran, Iran \\ 4. Associate professor of Cardiology, Shahid Rajaei Heart Hospital, Iran University of Medical \\ Sciences, Tehran, Iran \\ 5. Research Associate, Cardiology Ward, Tehran University of Medical Sciences, Tehran, Iran \\ 6. Internist, Department of Internal Medicine, Iran University of Medical Sciences, Tehran, Iran
}

\section{Corresponding Author:}

- Name: Morteza Hassanzadeh

- Address: Rasoul-e-Akram Medical Center, Niyayesh Street, Sattarkhan Ave., Tehran, Iran

- Telephone number: (+98) 9111376265

- FAX number: +982188925874 , +982188915410

- E-mail: Morteza hb@yahoo.com

\section{Short Title:}

BNP \& renovascular HTN

\section{Invitation/request to submit the manuscript:}

None

\section{KEYWORDS}

BNP, Hypertension, Renal artery stenosis, Angioplasty

Word count : 2650

Number of tables : 3

Number of figures : 1

Number of supplementary digital content files : 0 


\section{ABSTRACT}

Renal artery stenosis (RAS) is the most prevalent cause of secondary hypertension (HTN). Percutaneous trans-luminal renal angioplasty (PTRA) is used for both diagnosis and treatment of RAS. As many as one third of RAS cases fail to demonstrate post-PTRA HTN improvement. In this study, brain natriuretic peptide (BNP) was measured for consecutive patients with refractory HTN referred for renal artery angiography and 2 groups of participants (essential HTN and RAS) were compared. BNP was found significantly higher among RAS group than those with essential HTN $(P<0.001)$ and had a good sensitivity $(76 \%)$ to discriminate RAS from essential HTN. RAS cases also showed a statistically significant decline in BNP level $(P<0.001)$ after PTRA. We concluded that BNP is increased in RAS before intervention, is declined after PTRA, and might be used both as a marker for discriminating RAS from essential refractory HTN and for postPTRA follow-up.

\section{KEYWORDS}

BNP, Hypertension, Renal artery stenosis, Angioplasty

\section{Comments:}

$\checkmark \quad$ This work has not been previously presented elsewhere.

$\checkmark \quad$ All expenses of this study were provided by a grant from Iran University of Medical Sciences.

$\checkmark$ The authors declare that there have been no potential conflicts of interest. 


\section{INTRODUCTION}

\begin{abstract}
Hypertension (HTN) is a worldwide crisis. Statistics demonstrate that approximately $30 \%$ of the adult population in developed countries suffers from high blood pressure, among whom about $20 \%$ are not aware of the problem [1]. Although most of the patients are categorized as essentialtype HTN, some known conditions (renovascular disease, primary hyperaldosteronism, pheochromocytoma, obstructive sleep apnea, etc.) are responsible for the remaining $5-10 \%$ of cases - the secondary HTN group [2]. Nowadays, secondary HTN has been encountered with increasing frequency, and among all etiologies, renal artery stenosis (RAS) remains as the most prevalent and important cause that can make HTN resistant to standard treatment and is associated with accelerated target organ damage [3].
\end{abstract}

RAS is commonly detected by available vascular imaging modalities (such as duplex ultrasonography, magnetic resonance angiography or computed tomographic angiography) and its main targeted treatment, contemporarily with angiotensin converting enzyme inhibitor (ACEI) and other medications, is percutaneous trans-luminal renal angioplasty (PTRA). However, as many as one third of patients fail to demonstrate improvement in HTN after the procedure [4]. Looking for specific biomarkers to identify patients with good response to angioplasty, therefore, is of great importance to improve costeffectiveness of the procedure.

B-type natriuretic peptide (BNP) is a neuro-hormone that is mainly released from the heart and, as a hormonal link between the two organs, exerts diuretic and natriuretic effects on the kidney [5]. In contrast to physiologic state where there is an inverse relationship between plasma levels of renin and BNP, in pathologic situations such as heart failure and RAS both renin and BNP levels are increased [6]. Moreover, in-vitro experimental studies demonstrate that BNP mRNA is upregulated after inducing renovascular HTN by clipping the renal artery [5]. Therefore, BNP levels theoretically can be used as both an indicator of RAS and a marker of successful PTRA.

We designed this study to investigate whether plasma BNP level is specifically increased in patients with RAS (when compared to the essential cases of HTN), and whether BNP level is affected by PTRA.

\section{MATERIALS AND METHODS}

\subsection{Study Participants}

The study was conducted in the Shahid-Rajaei Heart Center, the biggest cardiology referral hospital in Tehran, Iran. From January 2013 until October 2014, all consecutive subjects referred for renal artery angiography were recruited. Excluded were those with congestive heart failure, valvular heart diseases, recent $(<6$ months) event of acute coronary syndrome, documented pulmonary emboli, pulmonary HTN, corpulmonale, hypo- or hyper-thyroidism, and chronic kidney disease with creatinine $>2$ $\mathrm{mg} / \mathrm{dl}$. Moreover, patients who revoke their consent at any stage of the study and those with any new confounding event (acute coronary syndrome, atheroembolic renal disease, acute kidney injury, etc.) were excluded.

\subsection{Study Protocol}

Recruited patients were hospitalized prior to the angiography if they were under ACEI, beta-blocker, or diuretic. Then they were included only if we could balance the treatment by reduction of beta blockers to at least $25 \%$ of their daily use, and cessation of 
diuretics and ACEIs for at least 3 days prior to the intervention.

All patients' information were collected and recorded before any treatment and intervention. The demographic data such as age and sex were retrieved from their medical files; and blood pressures were recorded using Riester sphygmomanometer (Riester GmbH., Jungingen, Germany). A 10 cc venous blood sample was taken from all subjects and levels of serum sodium, potassium, and creatinine were measured by routine kits (Pars Azmoon Co., Tehran, Iran). BNP level was analyzed using the Architect BNP immunoassay (Abbott Laboratories, Abbot Park, Illinois) with $4.2 \%$ intra-assay coefficient of variation at $94 \mathrm{pg} / \mathrm{mL}$. Also, all included cases underwent transthoracic echocardiography (Vivid 7 GE System) by a single echocardiography fellow.

All angiographic studies were performed by a single interventional cardiologist (after at least 3 days of admission for hospitalized subjects). Patients were categorized as "case" group if $>70 \%$ renal artery occlusion was seen; all of whom then underwent PTRA using drugeluted stents at the same session. Those without renal occlusion of $70 \%$ or more were considered as "control" group if we had a sex and age matched subject in the case group. Subjects of the case group were evaluated for BNP and creatinine one week after PTRA. The control group subjects received standard treatment according to recommendations of the American Heart Association. Blood pressures were also recorded after treatments in both groups.
The protocol of this study was approved by the ethics committee of the Iran University of Medical Sciences and all patients signed written informed consent.

\subsection{Statistical analysis}

All information was coded and entered into SPSS for Windows version 20.0. Results were expressed as mean and standard deviation (SD), median and range, or number and percentage as appropriate. Correlations between the data were tested using Pearson's coefficient. Comparison of data between the two groups was assessed by the t test and the Pearson chi-square test. The receiver operating characteristic (ROC) curves were derived to find the best cut-off point for BNP and other probably predictive factors. All statistical tests were two tailed, and a $\mathrm{P}$ value of 0.05 or less was considered as statistically significant.

\section{RESULTS}

After considering the inclusion and exclusion criteria for near 300 referral patients, 67 were included in the study, consisting of 17 cases and 50 controls. Baseline characteristics of the study subjects are shown in Table 1. As seen, systolic and diastolic blood pressures $(\mathrm{P}=0.003$ and $\mathrm{P}$ $<0.001$, respectively) and levels of serum creatinine $(\mathrm{P}<0.001)$ and $\mathrm{BNP}(\mathrm{P}<0.001)$ differ significantly between the two groups. None of the other comparisons demonstrated any meaningful difference. Also, no significant difference was found in the mean age between the genders $(\mathrm{P}=0.80)$. For all subjects, the plasma values of sodium, potassium, and creatinine were all within the reference range. 
Table 1. Baseline characteristics of the study subjects*

\begin{tabular}{lccc}
\hline & $\begin{array}{c}\text { Essential HTN } \\
(\mathbf{N}=\mathbf{5 0})\end{array}$ & $\begin{array}{c}\text { Renovascular HTN } \\
(\mathbf{N}=\mathbf{1 7})\end{array}$ & $\boldsymbol{P}$ \\
\hline Age (years) & $66 \pm 9$ & $66 \pm 9$ & 0.932 \\
Male Sex & $19(38)$ & $10(59)$ & 0.441 \\
Ejection Fraction (\%) & $50 \pm 4$ & $51 \pm 5$ & 0.561 \\
LV-End Diastolic Diameter (cm) & $4.7 \pm 0.6$ & $4.8 \pm 0.5$ & 0.658 \\
LV-End Systolic Diameter (cm) & $3.5 \pm 0.6$ & $3.4 \pm 0.4$ & 0.752 \\
Septum Diameter (mm) & $8.8 \pm 1.8$ & $8.4 \pm 2.1$ & 0.540 \\
LV Hypertrophy (yes) & $12(24)$ & $6(35)$ & 0.909 \\
SBP (mmHg) & $162 \pm 13$ & $155 \pm 10$ & 0.003 \\
DBP (mmHg) & $75 \pm 9$ & $86 \pm 9$ & $<0.001$ \\
Sodium (mEq/L) & $140 \pm 2$ & $140 \pm 4$ & 0.999 \\
Potassium (mEq/L) & $4.0 \pm 0.3$ & $4.0 \pm 0.3$ & 0.190 \\
Creatinine (mg/dL) & $0.8 \pm 0.2$ & $1.0 \pm 0.2$ & $<0.001$ \\
BNP (pg/mL) & $290.7 \pm 420.5$ & $837.1 \pm 649.8$ & $<0.001$ \\
\hline
\end{tabular}

* Values are the mean \pm standard deviation, or Number (\%) as appropriate; HTN: Hypertension; LV: Left Ventricle; SBP: Systolic Blood Pressure; DBP: Diastolic Blood Pressure

In ROC analysis, the area under the curve was $0.758(\mathrm{P}=0.02)$ (Figure 1). The best cut-off point for BNP was found to be
$312 \mathrm{pg} / \mathrm{mL}$ for discriminating essential from renovascular HTN with $76 \%$ sensitivity and $74.0 \%$ specificity (table 2 ).

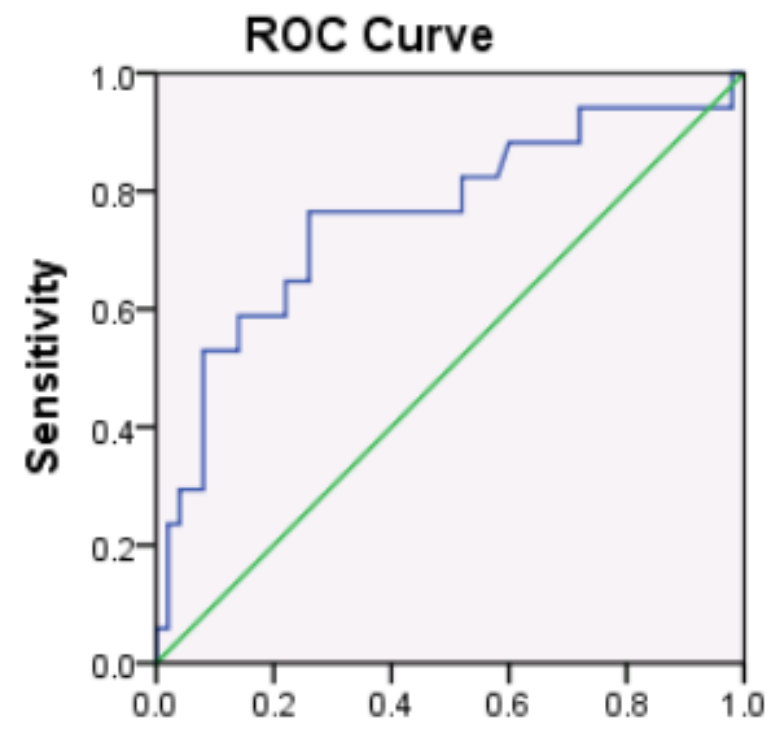

Figure 1. Receiver Operating Characteristic (ROC) analysis curve 
Table 2. Test characteristics of different BNP cutoffs for discriminating essential hypertension from renovascular hypertension based on the Receiver Operating Characteristic (ROC) analysis

\begin{tabular}{ccc}
\hline NP Cutoff $(\mathbf{p g} / \mathbf{m L})$ & Sensitivity (\%) & Specificity (\%) \\
\hline 258 & 76 & 68 \\
284 & 76 & 70 \\
299 & 76 & 72 \\
312 & 76 & 74 \\
315 & 71 & 74 \\
\hline
\end{tabular}

Intra-group comparisons for case group before and after PTRA are summarized in Table 3. As seen, levels of systolic and diastolic blood pressures $(\mathrm{P}<0.001)$, creatinine $(\mathrm{P}<0.001)$ and BNP $(\mathrm{P}<0.001)$ dropped significantly after PTRA.

Table 3. Comparison of measures for patients with renal artery stenosis before and after percutaneous transluminal renal angioplasty (PTRA)*

\begin{tabular}{lccc}
\hline & Before PTRA & After PTRA & $P$ \\
\hline SBP (mmHg) & $155 \pm 10$ & $140 \pm 10$ & $<0.001$ \\
DBP (mmHg) & $86 \pm 9$ & $78 \pm 8$ & $<0.001$ \\
Sodium (mEq/L) & $140 \pm 4$ & $140 \pm 2$ & 0.999 \\
Potassium (mEq/L) & $4.0 \pm 0.3$ & $4.0 \pm 0.3$ & 0.745 \\
Creatinine (mg/dL) & $1.0 \pm 0.2$ & $0.9 \pm 0.2$ & $<0.001$ \\
BNP (pg/mL) & $837.1 \pm 649.8$ & $438.2 \pm 339.2$ & $<0.001$ \\
\hline
\end{tabular}

* Values are the mean \pm standard deviation; SBP: Systolic Blood Pressure; DBP: Diastolic Blood Pressure

Further analysis demonstrated that systolic and diastolic blood pressure changes did not have any significant correlation with BNP changes. $(\mathrm{P}>0.05, \mathrm{r}<0.01$ for both measurements).

\section{DISCUSSION}

Based on the results of the present study, lower SBP, higher DBP, and higher levels of creatinine and BNP were found 
among patients with renovascular HTN when compared to those with essential HTN. Furthermore, patients with RAS showed a statistically significant decline in blood pressure (SBP and DBP) and the levels of creatinine and BNP after PTRA.

According to the literature, onset of severe HTN at >55 years of age, and accelerated, resistant, or malignant HTN are among clinical clues for the presence of RAS [7]. So without taking other clinical indices into account, the mere SBP or DBP level could not be considered as a distinct indicator of RAS. Therefore, the observed difference in SBP and DBP among the 2 groups is probably an incidental finding in our sample. Moreover, when all of the values are ranged within the normal limits, a statistical difference of about $0.2 \mathrm{mg} / \mathrm{dL}$ for serum creatinine is not usually considered as clinically meaningful.

Plasma BNP concentration has previously been shown to be elevated in patients with essential HTN [8-10]. There is a substantial overlapping, however, in BNP level between normotensive and hypertensive individuals $[9,11,12]$. Also, interestingly, in one study [13], higher plasma BNP concentration in nonhypertensive subjects has been associated with a greater likelihood of future HTN. In the present study, the mean BNP level was found significantly higher in patients with RAS than in patients with essential HTN. That finding would be best described by considering the pathophysiologic action of BNP in relation to the renin-angiotensinaldosterone system. It means that increased activity of the renin-angiotensin-aldosterone system, resulting from RAS, increases the secretion of BNP which opposes the effects of that system by promoting natriuresis [14].

To our knowledge, very few investigations as yet have been focused on comparing BNP level between patients with
RAS and those with essential HTN, and on looking for a BNP cut-point to discriminate RAS from essential HTN. Mussalo et al [15], in accordance to our finding, demonstrated higher mean BNP level among subjects with renovascular HTN. Their discriminative BNP cutoff $(9.8 \mathrm{pmol} / \mathrm{L}$ [33.91 $\mathrm{pg} / \mathrm{mL}]$ ), however, had lower sensitivity $(58 \%$ vs. $76 \%)$ and higher specificity ( $90 \%$ vs. $74 \%)$ than ours. Of note, in that study, the mean BNP level as well as the best BNP cutoff, both of which being within the reference range, have been reported as about 10 times lower values than our ones. Those significantly different achieved values could be attributed to the different populations studied and the relatively small sample size of both surveys. Yet, when considering better performance of our cutoff in terms of sensitivity, one possible hypothesis for future studies might be that if the BNP level in a given subject with HTN is above the normal limit, there is a certain BNP cutoff that would be sensitive enough to screen cases of renovascular HTN.

The finding that BNP level had a significant decline after PTRA is strongly in favor of a causal relationship between RAS and the rise of BNP. Since we excluded most other confounding circumstances (such as heart failure, acute coronary syndrome, etc.), the baseline BNP elevation in our study subjects could not be attributed to those conditions; and the post-PTRA BNP decline is more likely to be exclusively related to the successful revascularization. A similar finding has recently been reported by Silva et al [16]. Additionally, elevated BNP level (> $80 \mathrm{pg} / \mathrm{mL}$ ) in $82 \%$ (14 out of 17) of our subjects with RAS supports the animal study by Kurtz et al [5] in which the BNP mRNA is up regulated in the "two-kidney, one-clip" RAS model.

Finally, compared to results of some previous studies with failure rate of as high 
as $30 \%$ in HTN improvement after PTRA [17-19], we found a statistically significant decline in both SBP and DBP in our sample. As recurrence of HTN after initial improvement (post-PTRA) is unusual $[20,21]$, we had the highest post-procedural clinical success rate in the present study. Making direct comparisons among different investigations, however, is limited because of differences in participants' antihypertensive medications, target blood pressure, and defined criteria for improvement [4].

This was a prospective case-control study in which the potential confounding role of many clinical factors was minimized by defining some strict exclusion criteria. We also omitted any intra-observer variabilities by using a single interventionist for all PTRAs, and a single cardiologist for all echocardiographic evaluations. This study, however, is limited by the relatively small number of participants that increases the likelihood of type I statistical error.

In summary, we demonstrated that plasma BNP concentration in patients with RAS is higher than those with essential refractory HTN and that the elevated BNP level falls significantly after PTRA. Moreover, BNP has a good sensitivity to discriminate RAS from essential HTN.

\section{DISCLOSURE}

All of the expenses of this study were provided by a grant from the Iran University of Medical Sciences. The authors declare that there is no conflict of interest. 


\section{REFERENCES}

1. Yoon SS, Ostchega Y, Louis T. Recent trends in the prevalence of high blood pressure and its treatment and control, 1999-2008. NCHS Data Brief. 2010; 48:1-8.

2. Pullalarevu R, Akbar G, Teehan G. Secondary hypertension, issues in diagnosis and treatment. Prim Care. 2014; 41:749-64.

3. Textor SC. Secondary hypertension: renovascular hypertension. J Am Soc Hypertens. 2014; 12:943-5.

4. Safian RD, Textor SC. Renal-artery stenosis. N Engl J Med. 2001; 344:43142.

5. Wolf $\mathrm{K}$, Kurtz A, Pfeifer $\mathrm{M}$, et al. Different regulation of left ventricular ANP, BNP and adrenomedullin mRNA in the two-kidney, one-clip model of renovascular hypertension. Pflugers Arch. 2001; 442:212-7.

6. Demerath T, Staffel J, Schreiber A, et al. Natriuretic peptides buffer renindependent hypertension. Am J Physiol Renal Physiol. 2014; 306:F1489-98.

7. White CJ, Jaff MR, Haskal ZJ, et al. Indications for renal arteriography at the time of coronary arteriography: a science advisory from the American Heart Association Committee on Diagnostic and Interventional Cardiac Catheterization, Council on Clinical Cardiology, and the Councils on Cardiovascular Radiology and Intervention and on Kidney in Cardiovascular Disease. Circulation. 2006; 114:1892-1895.

8. Kohno M, Horio T, Yokokawa K, et al. Brain natriuretic peptide as a cardiac hormone in essential hypertension. Am J Med 1992; 92:29-34.
9. Buckley MG, Markandu ND, Miller MA, et al. Plasma concentrations and comparisons of brain and atrial natriuretic peptide in normal subjects and in patients with essential hypertension. $\mathbf{J}$ Hum Hypertens 1993; 7:245-250.

10. Irzmański R, Banach M, Piechota M, et al. Atrial and brain natriuretic peptide and endothelin-1 concentration in patients with idiopathic arterial hypertension: the dependence on the selected morphological parameters. Clin Exp Hypertens. 2007; 29:149-64.

11. Cheung BM, Brown MJ: Plasma brain natriuretic peptide and $\mathrm{C}$-type natriuretic peptide in essential hypertension. $\mathrm{J}$ Hypertens 1994; 12: 449-454.

12. Takeda T, Kohno M: Brain natriuretic peptide in hypertension. Hypertens Res 1995; 18:259-266.

13. Michael H. Freitag, Martin G. Larson, Daniel Levy, et al. Plasma Brain Natriuretic Peptide Levels and Blood Pressure Tracking in the Framingham Heart Study. Hypertension. 2003; 41:978-83.

14. Holmes SJ, Espiner EA, Richards AM, et al. Renal endocrine and hemodynamic effects of human brain natriuretic peptide in normal man. J Clin Endocrinol Metab. 1993; 76:91-96.

15. Mussalo H, Vanninen E, Ikäheimo R, et al. NT-proANP and BNP in renovascular and in severe and mild essential hypertension. Kidney Blood Press Res. 2003; 26:34-41.

16. Silva JA, Chan AW, White CJ, et al. Elevated brain natriuretic peptide predicts blood pressure response after stent revascularization in patients with 
renal artery stenosis. Circulation. 2005 Jan; 111(3):328-33.

17. Rocha-Singh KJ, Mishkel GJ, Katholi $\mathrm{RE}$, et al. Clinical predictors of improved long-term blood pressure control after successful stenting of hypertensive patients with obstructive renal artery stenosis. Cathet Cardiovasc Intervent. 1999; 47:167-172.

18. Radermacher J, Chavan A, Bleck J, et al. Use of Doppler ultrasonography to predict the outcome of therapy for renalartery stenosis. N Engl J Med. 2001; $344: 410-417$.
19. Lederman RJ, Mendelsohn FO, Santos $\mathrm{R}$, et al. Primary renal artery stenting: characteristics and outcomes after 363 procedures. Am Heart J. 2001; 42:314 323.

20. Ramsay LE, Waller PC. Blood pressure response to percutaneous transluminal angioplasty for renovascular hypertension: an overview of published series. BMJ 1990; 300:569-572

21. Libertino JA, Beckmann CF. Surgery and percutaneous angioplasty in the management of renovascular hypertension. Urol Clin North Am 1994; 21:235-243 